\title{
Patient Satisfaction with Medicare Annual Wellness Visits Administered by a Clinical Pharmacist Practitioner
}

\author{
Christina H. Sherrill, PharmD, BCACP; Jamie Cavanaugh, PharmD, CPP, BCPS; \\ and Betsy Bryant Shilliday, PharmD, CPP, BCACP
}

\begin{abstract}
BACKGROUND: In accordance with the Patient Protection and Affordable Care Act, Medicare provides coverage for annual wellness visits (AWVs) to eligible beneficiaries, which focus on preventative services, furnish personalized preventative health plans, and direct appropriate referrals. These visits may be conducted by a physician or another licensed practitioner working under the direct supervision of a physician. In North Carolina, pharmacists licensed as clinical pharmacist practitioners (CPPs) may perform and bill for AWVs, but there are limited data on patient satisfaction with pharmacists serving in this advanced role.
\end{abstract}

OBJECTIVES: To (a) investigate patient satisfaction with and perception of an initial Medicare AWV administered by a CPP in an academic internal medicine clinic and (b) examine the relationship between patient satisfaction and the number of interventions or referrals made during an AWV.

METHODS: All established patients of an academic internal medicine clinic aged 66 years and older were eligible for AWVs with a CPP, and those completing an AWV with a CPP were eligible for inclusion in this study. Patient satisfaction with the CPP and AWV was assessed by a telephone satisfaction questionnaire administered after the visit by a pharmacy student not affiliated with the clinic.

RESULTS: Forty-six patients were included in this study. Patients rated their satisfaction with the CPP at a mean of 4.7 and with the visit at a mean of 4.6 on a Likert scale ranging from 1 to 5 , where 1 was "very dissatisfied" and 5 was "very satisfied." Using a Likert scale of 1 to 5 , where 1 was "strongly disagree" and 5 was "strongly agree," patients responded with a mean of 4.4 that they were comfortable discussing their health information with a CPP and a mean of 4.1 that they were just as comfortable discussing their health information for this visit with a CPP as with their primary care physician.

CONCLUSIONS: Overall, patients were very satisfied with the CPP and AWVs and felt comfortable with CPPs as the providers of this service. As such, this study demonstrates that through Medicare AWVs, pharmacists can provide direct patient care and contribute to team-based initiatives.

J Manag Care Spec Pharm. 2017;23(11):1125-29

Copyright @ 2017, Academy of Managed Care Pharmacy. All rights reserved.

\section{What is already known about this subject}

Medicare provides coverage for annual wellness visits (AWVs) to eligible beneficiaries, which focus on preventative services, furnish personalized preventative health plans, and direct appropriate referrals.

- Medicare AWVs may be conducted by a physician or by another licensed practitioner working under the direct supervision of a physician

In North Carolina, pharmacists licensed as clinical pharmacist practitioners (CPPs) may perform and bill for AWVs.

\section{What this study adds}

This study demonstrates that patients are satisfied and comfortable with AWVs conducted by CPPs.

This study supports the existing body of literature and increases the generalizability of the results, since it was conducted in a different setting with a different patient population than previous studies. This study investigates the relationship between patient satisfaction and the number of interventions or referrals made during an AWV.

$\bigcirc$ ince January 1, 2011, Medicare has offered beneficiaries full coverage for completing annual wellness visits (AWVs) in accordance with the Affordable Care Act. ${ }^{1}$ AWVs are outpatient appointments focused on health promotion and preventative services. Patients are eligible for an initial AWV after receiving Medicare Part B coverage for at least 12 months, and subsequent AWVs are covered by Medicare when separated by at least 12 months from the initial and any subsequent AWVs. The provision of an initial AWV includes administering and reviewing a health risk assessment; establishing a list of current providers and the beneficiary's medical and family history; assessing home safety, fall risk, hearing impairment, ability to perform activities of daily living, cognition, and depression risk; measuring height, weight, body mass index, and blood pressure; conducting other pertinent routine measurements according to medical and family history; and referring patients appropriately. Subsequent AWVs are less extensive and are not discussed here. ${ }^{1,2}$

AWVs may be conducted by "a physician, qualified nonphysician practitioner, medical professional (including a health educator, registered dietitian, nutrition professional, or other 
licensed practitioner), or team of such medical professionals who are working under the direct supervision of a physician."1,2 This policy is different from the policy for initial preventive physical examinations (IPPEs), which are one-time visits for Medicare Part B beneficiaries within the first 12 months of coverage that must be provided by physicians, physician assistants, nurse practitioners, or certified clinical nurse specialists. ${ }^{3}$ As Medicare AWVs have become more widespread, physicians have been looking to other members of the health care team to aid in the AWV workload, which can be time consuming and burdensome. ${ }^{4}$

In North Carolina, a pharmacist with prespecified training and qualifications may apply with the state board of pharmacy and medical board to become a licensed clinical pharmacist practitioner (CPP). CPPs in North Carolina maintain collaborative practice agreements (CPAs) with physicians and may perform and bill for services specified in the CPA, including AWVs, if "incident to" rules are met. ${ }^{5}$

Several studies have investigated the implementation process, resulting interventions, and financial considerations of Medicare AWVs that have been conducted by pharmacists. ${ }^{6-9}$ One such study, by Sewell et al. (2016), demonstrated that the quantity of interventions made by a pharmacist conducting AWVs was comparable to AWVs performed by physicians with similar reimbursement rates. ${ }^{6}$ This was similar to our previous findings, which indicated an average of 4.5 interventions per AWV administered by a CPP. ${ }^{9}$ However, data regarding patient satisfaction with and perception of pharmacist-administered AWVs are limited. In another study, patients in a family medicine practice were satisfied with pharmacist-led AWVs. ${ }^{10}$

The purpose of our study was to add to the currently available literature, thereby increasing the confidence of reproducibility, by describing the feedback received from patients via a telephone questionnaire regarding their satisfaction with AWVs and the CPPs who conducted them. In addition, this study examined the relationship between patient satisfaction and the number of interventions or referrals made during the AWV visit.

\section{Methods}

This study was conducted between June 2012 and March 2013 at an internal medicine clinic within an academic medical center. Patients were included if they were an established patient in the clinic (defined as a patient assigned to a primary care physician in the clinic and seen within the previous 18 months), aged at least 66 years, had been seen by a CPP for an initial Medicare AWV, and provided either written or verbal consent for study participation.

The logistics of enrolling patients for initial AWVs within the internal medicine clinic have been described elsewhere. ${ }^{9}$ In short, at the time of this study, established clinic patients eligible for an AWV were mailed invitations to call and schedule an AWV appointment. Once patients called and scheduled their AWVs, they were mailed health risk assessment packets to complete before the scheduled appointments. This packet included validated assessment tools for the various required elements of the AWV and was used to guide the AWV and determine which interventions were most appropriate for each patient. The assessment tools included HHIE-S (hearing), ${ }^{11}$ PHQ-9 (depression), ${ }^{12}$ AUDIT-C (alcohol), ${ }^{13}$ Timed Up and Go test (fall risk), ${ }^{14} 6$-item Cognitive Screener (dementia), ${ }^{15}$ screening questions based on the American Geriatrics Society/British Geriatrics Society Clinical Practice Guideline for Prevention of Falls in Older Persons (home safety) ${ }^{16}$ and screening questions based on the 4-year Mortality Index (functional status). ${ }^{17}$

Interventions and referrals are made according to evidencebased protocols established by the clinic based on results of the validated screening tools and recommendations from the U.S. Preventive Services Task Force. The CPP can also recommend, initiate, and adjust medications consistent with the CPP's scope of practice and CPA, as clinically indicated. As required by the Centers for Medicare \& Medicaid Services, a personalized prevention plan is provided to each patient at the conclusion of the visit to summarize interventions and referrals made during the AWV, as well as an outline of future treatment plans related to preventative care.

This study was conducted in 2 phases in order to address the 2 study objectives.

\section{Phase 1}

Phase 1 included a 17-question self-designed telephone questionnaire to assess patient opinions of recent AWVs. The questionnaire items addressed general satisfaction and satisfaction with the CPP and AWV, using Likert scales, multiple choice questions, and open-ended questions. The items most relevant to this study are shown in Table 1 . To limit recall bias, patients were called within 2 weeks of their AWVs and pursued until contact was made and either informed consent received and the questionnaire completed, or the patient declined enrollment. To limit potential reporting bias, all questionnaires were administered by a single pharmacy student rather than the CPP or an associated employee of the clinic. The pharmacy student was a volunteer matched to this research opportunity and did not work with the clinic in any other capacity.

\section{Phase 2}

Phase 2 consisted of a retrospective chart review to determine the interventions made by the CPP during the AWVs. The chart review was conducted by the pharmacy student, using a standardized spreadsheet developed in conjunction with the study team before the initiation of the study. Possible interventions included, but were not limited to, providing education regarding home safety or advanced care planning; recommending/ ordering laboratory tests or vaccination administration; referral for "local counseling on aging," physical therapy, nutrition, audiology, or colon cancer screening, including colonoscopy; and recommending changes to medication regimens. 
TABLE 1 Patient Satisfaction Survey Results ( $N=46$ )

\begin{tabular}{|c|c|c|c|}
\hline $1=$ very dissatisfied $; 5=$ very satisfied & Mean & Median & Range \\
\hline How would you rate the care you received from the provider? & 4.7 & 5 & $3-5$ \\
\hline How would you rate your satisfaction with the visit? & 4.6 & 5 & $2-5$ \\
\hline 1 = strongly disagree; 5 = strongly agree & Mean & Median & Range \\
\hline I am comfortable discussing my health information with a clinical pharmacist. & 4.4 & 4 & $3-5$ \\
\hline I am just as comfortable discussing my health information for this visit with a clinical pharmacist as with my PCP. & 4.1 & 4 & $2-5$ \\
\hline I would rather see my PCP for this visit. & 3.0 & 3 & $1-5$ \\
\hline I will repeat this visit each year. & 4.0 & 4 & $2-5$ \\
\hline I would like to see the same provider next year for a Medicare annual wellness visit. & 4.1 & 4 & $2-5$ \\
\hline This visit was important for my overall health. & 3.9 & 4 & $2-5$ \\
\hline This visit led me to make a change in my behavior to improve my health. & 3.4 & 4 & $2-5$ \\
\hline
\end{tabular}

$P C P=$ primary care provider

Both phases of this study were approved by the institutional review board. Descriptive statistics were used for data analysis.

\section{Results}

$\overline{\text { Fifty-one patients met inclusion criteria and provided consent. }}$ Five patients were unavailable by telephone, leaving 46 patients included in this study. The patient population was $52 \%$ female, $87 \%$ Caucasian, and $73.6 \pm 6.3$ years old (mean \pm standard deviation [SD]). Fifty-seven percent of patients had hypertension; $43 \%$ had dyslipidemia; $17 \%$ had diabetes; and $11 \%$ had coronary artery disease. The body mass index was $27.4 \pm 5.0$ $\mathrm{kg} / \mathrm{m}^{2}$ (mean $\pm \mathrm{SD}$ ). Fifty-seven percent were former smokers; $39 \%$ were never smokers; and 4\% were current smokers. Visits for all 46 patients were conducted by the same CPP, except for 1 that was conducted by a postgraduate year 2 pharmacy resident under the direct supervision of the CPP who conducted all the other AWVs.

The average time from AWV to first call attempt and questionnaire completion was 7.4 days (median 7 days) and 13 days (median 9 days), respectively. Every effort was made to contact each patient within 2 weeks of the first visit, and all questionnaires were completed within 2 months of the AWV. No patients declined study participation once contact was made.

\section{Phase 1}

Results from Phase 1 are presented in Table 1. Seventy-two percent of patients answered that they were "very satisfied" with the care received from the CPP, and 70\% were "very satisfied" with the visit as a whole. Ninety-eight percent of patients either "agreed" or "strongly agreed" that they were comfortable discussing their health information with a CPP, and $87 \%$ either "agreed" or "strongly agreed" that they were just as comfortable discussing their health information for this visit with a CPP as with their primary care physician (PCP) and that they would like to see the same provider next year for their AWV.

When asked if they would rather see their PCP for the AWV, $35 \%$ of patients "agreed" or "strongly agreed," while $44 \%$ of patients "disagreed" or "strongly disagreed." Patient comments were recorded regarding this question, and in general, patients favoring the CPP conducting the AWVs enjoyed getting a different and new perspective on their health, while those desiring to have their PCP administer the visit were comforted by the established relationship that they had with their PCP and that their PCP already knew them and their medical history.

\section{Phase 2}

Multiple interventions were made during each AWV with the CPP. Home safety education, which included education regarding emergency telephone numbers and the use of nonslip rugs to prevent falls, was the most common intervention, with $70 \%$ of patients receiving this intervention. Recommendations for the herpes zoster and tetanus vaccinations were also common, as was the provision of information about advanced care planning (41\%, 39\%, and 30\%, respectively).

The number of interventions for each level of patient satisfaction is presented in Table 2. On average, patients who were more satisfied with the CPP received a higher number of interventions during the visit. Patients who were "satisfied" or "very satisfied" with the AWV had more interventions than those who were "dissatisfied" or "neither satisfied nor dissatisfied" with the AWV.

\section{Discussion}

This study supports the existing evidence of patient satisfaction with a pharmacist conducting an AWV and increases the generalizability of the data by using a different patient population. Wilson et al. (2015) used a questionnaire and clinic process adapted from that which is described in this article. ${ }^{10}$ Overall, the patient satisfaction results of the current study were similar to the Wilson et al. study, adding to the reproducibility and validity of the results found by Wilson et al. ${ }^{10}$ Specifically, differences in mean responses were 0.1 on the 5-point Likert scale for patient responses regarding satisfaction with the CPP and with the visit. In addition, differences 


\begin{tabular}{|c|c|c|c|}
\hline \multirow[b]{2}{*}{ Satisfaction with CPP $(\mathrm{N}=46)$} & \multicolumn{3}{|c|}{ Number of Interventions } \\
\hline & Mean & Median & Range \\
\hline Very satisfied $(n=33)$ & 4.0 & 4 & $0-9$ \\
\hline Satisfied (n=12) & 3.8 & 3 & $2-8$ \\
\hline \multirow[t]{2}{*}{ Neither satisfied nor dissatisfied $(n=1)$} & 3.0 & 3 & 3 \\
\hline & \multicolumn{3}{|c|}{ Number of Interventions } \\
\hline Satisfaction with AWV $\left(\mathrm{N}=45^{\mathrm{a}}\right)$ & Mean & Median & Range \\
\hline Very satisfied $(n=32)$ & 4.2 & 4 & $2-8$ \\
\hline Satisfied $(n=8)$ & 4.4 & 3.5 & $3-9$ \\
\hline Neither satisfied nor dissatisfied $(n=4)$ & 1.8 & 2 & $0-3$ \\
\hline Dissatisfied $(\mathrm{n}=1)$ & 3.0 & 3 & 3 \\
\hline \multicolumn{4}{|c|}{$\begin{array}{l}\text { a One patient answered } 4.5 \text { versus } 4 \text { (satisfied) or } 5 \text { (very satisfied) so was excluded } \\
\text { from these results. } \\
\text { AWV = annual wellness visit: } C P P=\text { clinical pharmacist practitioner. }\end{array}$} \\
\hline
\end{tabular}

in mean responses were less than 0.5 on the 5-point Likert scale for patient responses regarding the level of comfort with discussing their health information with a CPP compared with their PCP, as well as whether or not they would rather see their PCP for the AWV visit. The greatest discrepancies in survey results between the current study and Wilson et al. were with regard to patients repeating the visit each year and the importance of the visit for their overall health (differences in mean responses of 0.9 , with the previous study showing more favorable results).

A possible reason for the variability in results could be disparities between the patient populations. The current study was conducted in a more urban area with an overall higher socioeconomic climate compared with the study by Wilson et al., which could cause discrepancies in access to health care. ${ }^{18}$ This disparity could explain why patients in the current study were not as enthusiastic about participating in subsequent AWVs and why the visits may not have been perceived as providing as great of a degree of benefit, indicating that the patients may be healthier overall. Furthermore, the current study did show a trend toward decreased patient satisfaction in patients who received fewer interventions or referrals.

Despite some differences between the current study and Wilson et al., both studies reveal high levels of patient satisfaction with AWVs and with CPPs administering the AWVs. Together, these studies support using pharmacists to conduct AWVs as part of the interdisciplinary health care team. Further research should be conducted to understand whether the perception of pharmacists performing Medicare AWVs influence a patient's decision to attend or decline an AWV. In addition, studies could be conducted to investigate long-term outcomes of AWVs conducted by CPPs, including the effect of interventions made by CPPs.

\section{Limitations}

Limitations of this study include the potential for recall bias, since the questionnaire was conducted after the visit via telephone versus in the clinic immediately following the visit, in an effort to increase response rates, as well as reduce reporting bias. This limitation was mitigated by multiple attempts to reach patients, with a mean successful questionnaire completion time of 13 days following the visit. In addition, there could be selection bias because the AWV invitations were only mailed out in English, which might indirectly exclude patients with low literacy or who do not speak English. This study also only included patients who opted-in to this health care service, which could add to selection bias.

This study did not examine the patients who did not schedule an AWV, so the difference between those patients that opted-in versus those who did not respond is not known. Furthermore, the route of questionnaire administration may prohibit the inclusion of patients who do not have a telephone, do not readily use a telephone, or have hearing impairment. Finally, because of the geographic location of the internal medicine clinic, the results of the study may have inadvertently been skewed toward healthier patients and those with a higher socioeconomic status, compared with the general population.

\section{Conclusions}

Patients were accepting of and very satisfied with CPPs conducting Medicare AWVs. In addition, patients who received more interventions generally reported a higher degree of satisfaction, suggesting the benefit of targeting patients with the potential for more interventions for providers wanting to initiate this service. Medicare AWVs offer an opportunity for pharmacists to provide direct patient care focused on preventative health, identify areas for patient interventions, and contribute to team-based care, and CPPs may serve as a viable option to expand patient access and care with the growing shortage of PCPs.

\section{Authors}

CHRISTINA H. SHERRILL, PharmD, BCACP, Eshelman School of Pharmacy, University of North Carolina, Chapel Hill. JAMIE CAVANAUGH, PharmD, CPP, BCPS, and BETSY BRYANT SHILLIDAY, PharmD, CPP, BCACP, Eshelman School of Pharmacy and School of Medicine, University of North Carolina, Chapel Hill.

AUTHOR CORRESPONDENCE: Christina H. Sherrill, PharmD, BCACP, Fred Wilson School of Pharmacy, High Point University, One University Pkwy, High Point, NC 27268. Tel.: 336.841.9678; E-mail: Csherril@highpoint.edu. 


\section{DISCLOSURES}

No outside funding supported this study, and the authors have no conflicts of interest to declare.

Study concept and design were contributed by Cavanaugh and Shilliday, along with Sherrill. The data were collected by Sherrill and Shilliday and interpreted by Sherrill, Cavanaugh, and Shilliday. The manuscript was written and revised by Sherrill, Cavanaugh, and Shilliday.

This work was presented at the ASHP Annual Meeting in Las Vegas, Nevada, on December 3, 2012.

\section{ACKNOWLEDGMENTS}

The authors acknowledge Keith Warshany, PharmD, PhC, BCPS, for his contributions to this study.

\section{REFERENCES}

1. Centers for Medicare \& Medicaid Services. Annual wellness visit (AWV), including personalized prevention plan services (PPPS). MLN Matters. Number: MM7079. January 2011. Available at: https://www.cms. gov/Outreach-and-Education/Medicare-Learning-Network-MLN/ MLNMattersArticles/downloads/mm7079.pdf. Accessed August 29, 2017.

2. Centers for Medicare \& Medicaid Services. The ABCs of the annual wellness visit (AWV). April 2017. Available at: https://www.cms.gov/Outreachand-Education/Medicare-Learning-Network-MLN/MLNProducts/downloads/ AWV_chart_ICN905706.pdf. Accessed August 29, 2017.

3. Centers for Medicare \& Medicaid Services. The ABCs of the initial preventive physician examination (IPPE). April 2017. Available at: https:// www.cms.gov/Outreach-and-Education/Medicare-Learning-Network-MLN/ MLNProducts/downloads/mps_qri_ippe00la.pdf. Accessed August 29, 2017.

4. Cuenca AE. Making Medicare annual wellness visits work in practice. Fam Pract Manag. 2012;19(5):11-16.

5. State of North Carolina. Clinical pharmacist practitioner. 21 NCAC 32T 010. April 2001. Available at: http://reports.oah.state.nc.us/ncac/title\%2021\%20 -\%20occupational\%20licensing\%20boards\%20and\%20commissions/ chapter\%2032\%20-\%20north\%20carolina\%20medical\%20board/subchapter\%20t/subchapter\%20t\%20rules.html. Accessed August 29, 2017.
6. Sewell MJ, Riche DM, Fleming JW, et al. Comparison of pharmacist and physician managed annual Medicare wellness services. J Manag Care Spec Pharm. 2016;22(12):1412-16. Available at: http://www.jmcp.org/ doi/10.18553/jmcp.2016.22.12.1412.

7. Park I, Sutherland SE, Ray L, et al. Financial implications of pharmacist-led Medicare annual wellness visits. J Am Pharm Assoc (2003). 2014:54(4):435-40.

8. Thomas MH, Goode JV. Development and implementation of a pharmacist-delivered Medicare annual wellness visit at a family practice office. J Am Pharm Assoc (2003). 2014;54(4):427-34.

9. Warshany K, Sherrill CH, Cavanaugh J, et al. Medicare annual wellness visits conducted by a pharmacist in an internal medicine clinic. Am J Health Syst Pharm. 2014;1(1):44-49.

10. Wilson CG, Park I, Sutherland SE, et al. Assessing pharmacist-led annual wellness visits: interventions made and patient and physician satisfaction. J Am Pharm Assoc (2003). 2015;55(4):449-54.

11. Ventry IM, Weinstein BE. Identification of elderly people with hearing problems. ASHA. 1983;25(7):37-42.

12. Kroenke K, Spitzer RL, Williams JB. The PHQ-9: validity of a brief depression severity measure. J Gen Intern Med. 2001;16(9):606-13.

13. Bush K, Kivlahan DR, McDonell MB, et al. The audit alcohol consumption questions (AUDIT-C): an effective brief screening test for problem drinking. Ambulatory care quality improvement project (ACQUIP). Alcohol use disorders identification test. Arch Intern Med. 1998;158(16):1789-95.

14. Podsiadlo D, Richardson S. The timed "UP \& Go": a test of basic functional mobility for frail elderly persons. J Am Geriatr Soc. 1991;39(2):142-48. 15. Callahan CM, Unverzagt FW, Hui SL, et al. Six-item screener to identify cognitive impairment among potential subjects for clinical research. Med Care. 2002;40(9):771-81.

16. Panel on Prevention of Falls in Older Persons, American Geriatrics Society and British Geriatrics Society. American Geriatrics Society/British Geriatrics Society clinical practice guideline for prevention of falls in older persons. J Am Geriatr Soc. 2011;59 (1):148-57.

17. Lee SJ, Lindquist K, Segal MR, et al. Development and validation of a prognostic index for 4-year mortality in older adults. JAMA. 2006;295(7):801-08.

18. U.S. Census Bureau. QuickFacts: North Carolina. Available at: https:// www.census.gov/quickfacts/fact/map/NC/INC110215\#viewtop. Accessed September 21, 2017. 